\author{
Anna Sulikowska \\ ORCID: 0000-0003-2939-3031 \\ Universität Szczecin, Szczecin
}

DOI: $10.19195 / 0435-5865.143 .23$

\title{
Metapher, Metonymie und mentales Bild als Motiviertheitsmechanismen in der Phraseologie
}

\begin{abstract}
s
Das Ziel des Artikels liegt in der Veranschaulichung der Komplexität von Bedeutungskonstituierungs- und Motiviertheitsmechanismen in der Phraseologie. In einer korpusbasierten semantischen Untersuchung des Idioms ein harter Brocken werden Verwendungsprofile ermittelt und kognitive Mechanismen aufgezeigt, die zur Konstruktion der Bedeutung führen und sie motivieren. Außer den etablierten Metaphern und Metonymien wird auch der Einfluss des mentalen Bildes als ein kognitiver Mechanismus aufgezeigt und diskutiert.
\end{abstract}

Schlüsselwörter: Phraseologie, Bedeutung, Metapher, Metonymie

\section{Metaphor, metonymy and rich image as motivating mechanisms in phraseology}

The aim of the article is to show the complexity of meaning construction and motivation procedures within phraseology. The research concentrates on the idiom ein harter Brocken, on the basis of which usage profiles and cognitive mechanisms have been shown, which support the construction of its meaning and motivation. Another research topic, beyond established metaphors and metonymies, was the influence of the mental image as a cognitive mechanism.

Keywords: phraseology, meaning, metaphor, metonymy

Anna Sulikowska, Uniwersytet Szczeciński, Instytut Filologii Germańskiej, Al. Piastów 40b, bud. 5, pok. 103, 71-065 Szczecin, Polen, E-Mail: ania.sulikowska@gmail.com

Received: 22.09.2017, accepted: 18.05.2018 
Schon in der Anfangsphase der wissenschaftlichen Beschäftigung mit der Phraseologie wurde darauf verwiesen, dass die Beschreibung der Bedeutung von Phraseologismen schwieriger als bei Einwortlexemen ist (Černyševa 1980: 44). Die meisten Phraseologismen im engeren Sinne (= Idiome) weisen nämlich Merkmale auf, deren Erfassung im Rahmen der traditionellen, d.h. strukturalistisch oder generativistisch geprägten Sprachwissenschaft äußerst problematisch ist: Sie sind motiviert und bildhaft.

Die Motiviertheit wird in der Phraseologie der Idiomatizität gegenübergestellt. Unter Idiomatizität versteht man die Unableitbarkeit der Bedeutung einer Wortverbindung aus den Bedeutungen ihrer Konstituenten. So lässt sich das Idiom jmdm. den Kopf waschen aus synchroner Perspektive kaum erklären: Weder der Kopf noch das Waschen tragen zur phraseologisierten Bedeutung ,jmdn. zurückweisen, tadeln ' bei. Die Beziehung zwischen der literalen und der phraseologisierten Lesart ist arbiträr, willkürlich, weswegen die Wortverbindung als hochgradig idiomatisch gilt - es ist ein sog. opakes Idiom. Sieht man aber prüfend die idiomatischen Wörterbücher durch, dann stellt sich heraus, dass nur wenige Idiome völlig idiomatisch sind, in den meisten Fällen lässt sich unter Rückgriff auf das Weltwissen eine Erklärung für die idiomatische Lesart finden. So ist der Phraseologismus jmdn. wie ein rohes Ei behandeln, jmdn. delikat, sanft behandeln' auf das Alltagswissen zurückzuführen, dass Eierschalen zerbrechlich sind. Beim Idiom wissen, wo jmdn. der Schuh drückt, die Probleme, Sorgen einer anderen Person kennen' greifen wir auf unangenehme und schmerzhafte körperliche Erfahrungen mit unbequemen Schuhen zurück und übertragen sie in die psychologische Sphäre. Die Verbindungen zwischen der literalen und der phraseologisierten Lesart sind für die meisten Sprecher leicht nachvollziehbar, die Idiome sind motiviert, auch wenn diese Motiviertheit nachträglich ist, d.h. die Zuweisung der Interpretation erfolgt, falls kein Kontext vorhanden ist, vor dem Hintergrund der bereits bekannten phraseologisierten Bedeutung. ${ }^{1}$

Die phraseologische Bildhaftigkeit beruht darauf, dass zahlreiche Idiome mentale Bilder evozieren können. Unter mentalen Bildern versteht man „konsistente mentale Abbildungen der externen Informationsbasis“ (Podding 1995: 176, zit. nach Stöckl 2004: 56). Die psychologische Realität der mentalen Bilder hat bereits der Vater der europäischen Phraseologie, Charles Bally, wahrgenommen, jahrelang blieb sie aber als sekundärer Phraseologizitätsindikator nur am Rande des sprachwissenschaftlichen Interesses.

${ }^{1}$ Wäre das Idiom jmdn. wie ein rohes Ei behandeln nicht lexikalisiert, hätte es keine konventionalisierte (phraseologisierte) Bedeutung, dann würde es wie eine beliebige ad hoc-Metapher funktionieren und man könnte es z.B. zweierlei interpretieren: 1. jmdn. stark verprügeln, brutal zusammenschlagen, so dass er zusammenbricht, 2. jmdn. sehr delikat behandeln, weil er so wie ein Ei sehr empfindlich ist. Die Tatsache, dass wir bei der Auslegung des Idioms nur auf die zweite Bedeutung zurückgreifen, ergibt sich daraus, dass wir diese phraseologisierte, konventionalisierte Bedeutung bereits kennen und nur diese Bedeutung uns den Interpretationsraum eröffnet. 
Die Tatsache, dass die Motiviertheit und Bildhaftigkeit bis vor Kurzem als problematische Phraseologizitätsmerkmale betrachtet wurden, hängt zweifelsohne mit der modularen Auffassung der Sprache in zwei einflussreichen linguistischen Theorien zusammen: dem Strukturalismus und dem Generativismus. Beide Theorien gehen von der strikten Abgrenzung des Sprachwissens von dem Weltwissen sowie der Kognition von der Perzeption aus: Der Rückgriff auf die allgemeinen Wissensstrukturen, der für die Motiviertheit unabdingbar ist, kann im Rahmen dieser Theorien also nicht erklärt werden. Im Generativismus wurde des Weiteren angenommen, dass das Sprachwissen ausschließlich in einer abstrakten, propositionalen Form mental repräsentiert ist: Dies stellt die Existenz der mentalen Bilder in Frage oder lässt sie bestenfalls als ein Epiphänomen der Kognition betrachten. Erst der holistisch geprägte Ansatz der Kognitiven Linguistik eröffnet einen Spielraum, in dem die phraseologische Motiviertheit und Bildhaftigkeit theoretisch verankert werden können. Mit der skizzenhaften Darstellung der Grundprämissen dieses Ansatzes wird der Beitrag eingeleiet.

\section{Grundprämissen des holistischen Ansatzes der Kognitiven Linguistik}

Die holistischen Grundannahmen lassen sich in folgenden Punkten zusammenfassen:

(i) Sprachliche Einheiten sind bipolar und symbolisch (Langacker 2008: 15-16), weil sie aus zwei Polen, einem phonologischen (oder graphematischen) und einem semantischen Pol bestehen, die durch eine symbolische, auf Konvention beruhende Beziehung verbunden sind. Als sprachliche Einheiten werden die Entitäten von unterschiedlicher Größe und Komplexität verstanden: Morpheme, Lexeme, Wortverbindungen.

(ii) Der semantische Pol ist nicht nur auf sprachliches Wissen (beispielsweise in Form von Merkmalslisten oder Netzwerken) eingeschränkt, sondern umfasst das ganze durch direkte Erfahrung erworbene oder vermittelte Wissen. Dies veranschaulicht Langacker (1987: 154) am Beispiel des Wortes banana. Die semantischen Merkmale, die einer Banane im Rahmen der Merkmalstheorie zugewiesen werden könnten (Obst, gelb, längliche Form, süß), sind für die Konzeptualisierung der Wortverbindung banana republic sekundär. Grundlegend für diese Konzeptualisierung ist ein eher peripheres Wissen über die Rolle der Bananen als einer Exportware für die Wirtschaft mancher kleiner, vor allem mittelamerikanischer Länder. Somit gibt es keine Abgrenzung zwischen dem linguistisch relevanten Sprachwissen und dem Weltwissen, die Bedeutungen sind holistisch, sie nehmen Bezug auf alle allgemeinen Weltwissensstrukturen. „Everything you know about the concept is part of its meaning" (Croft 2003: 163). 
(iii) In der neueren kognitiv ausgerichteten Forschung wird der Frage nach der Natur der mentalen Repräsentationen, die den semantischen Pol bilden, nachgegangen. Die im Rahmen der Kognitiven Linguistik angesiedelten Ansätze der Embodied/ Grounded Cognition liefern empirische Evidenz dafür, dass mentale Wissensrepräsentationen, die den konzeptuellen Pol einer sprachlichen Einheit konstituieren, modalitätsspezifisch sind, d.h. visuelle Reize als visuelle mentale Repräsentationen, auditive, taktile, gustatorische Reize in entsprechenden mentalen Modalitäten aufbewahrt werden. Die bisher vorausgesetzten abstrakten, amodalen Propositionen werden als eine mögliche, aber keinesfalls einzige Repräsentationsart angenommen. Eine kompakte Theorie über die mentalen modalitätsspezifischen Repräsentationen stellt Barsalou $(1999,2012)$ in seiner Perceptual Symbol System-Theorie dar, eine Übersicht über die Forschungsergebnisse, die diese Annahmen bestätigen, findet man in Rickheit/ Weiß/ Eikmeyer (2010: 108-115).

(iv) Die Bedeutungen sind dynamisch, gebrauchs- und erfahrungsbasiert: Sie entstehen in der Interaktion, on-line, sind emergent, werden unter sich ständig wechselnden kontextuellen Bedingungen immer neu ausgehandelt. Im Zentrum des linguistischen Interesses liegen dynamische Konzeptualisierungen, sie machen die Grundlage für die Herausbildung konventioneller Bedeutungen aus. Konzepte formen sich auf der Grundlage rekurrenter Sprachgebrauchsereignisse (Konzeptualisierungen), auf denen das kognitive Verfahren der Schematisierung operiert. Sie werden nicht als stabile Strukturen mit scharfen Grenzen, sondern als kognitive Routinen aufgefasst.

\section{Metapher und Metonymie als kognitive Motiviertheitsmechanismen}

Wie ersichtlich, stellt die Kognitive Linguistik einen theoretischen Rahmen dar, in dem die phraseologische Motiviertheit und Bildhaftigkeit angesiedelt werden können. Der Zugang zu allen, teilweise auf individueller Erfahrung beruhenden Wissensstrukturen eröffnet einen Spielraum für Assoziationen, Übertragungen aus dem einen in einen anderen Erfahrungsbereich, was für die phraseologische Motiviertheit konstitutiv ist. Als wichtigste Mechanismen der phraseologischen Motiviertheit werden Metapher und Metonymie (Burger 2007: 99-101; Palm 1995: 14) angesehen, die man aus kognitiver Perspektive folgendermaßen beschreiben kann:

Als Metapher wird ein kognitiver Mechanismus bezeichnet, in dem eine Erfahrungsdomäne mit einer anderen Erfahrungsdomäne (der Zieldomäne) durch konzeptuelle Mappings verbunden wird. Beide Domänen liegen in verschiedenen Erfahrungsbereichen, wobei die Ausgangsdomäne sich meistens auf eine kon- 
krete, körperliche Erfahrung bezieht, während die Zieldomäne abstrakter ist. So wird die höchst unangenehme körperliche Erfahrung oder die Vorstellung der Erfahrung von in die Zange genommen werden in die psychologische Sphäre des menschlichen Lebens übertragen und bezieht sich auf eine ausweglose, schwierige Lage, in der Druck von zwei Seiten ausgeübt wird. Metaphorische Mappings sind dynamisch, je nach dem Kontext werden bestimmte Aspekte hervorgehoben, andere treten in den Hintergrund. Die Metaphern, die auf Grund des Rückgriffs auf allgemeine Wissensstrukturen entstehen und auf einzelne Wortverbindungen beschränkt sind, bezeichne ich im Folgenden nach Barcelona (2003: 221) als epistemische Metaphern. Eine besondere Rolle kommt in der Kognitiven Linguistik den konzeptuellen Metaphern zu, die als körperlich verankerte Denkmodi aufgefasst werden. Konzeptuelle Metaphern sind kategorial, fest etabliert, sie entstehen nicht in der realen Sprechzeit, sondern werden als Strukturierungsprinzipien der menschlichen Kognition betrachtet, die sich in Sprache und Kultur manifestieren können (Lakoff/ Johnson 1980, Lakoff 1993, Kövecses 2002).

Die Metonymie beruht auf der Bedeutungsübertragung innerhalb einer Domäne (d.h. eines strukturierten Erfahrungsbereiches), wodurch der konzeptuelle Abstand zwischen den beiden Entitäten klein ist. Ein typisches Beispiel einer Metonymie liegt im Idiom pro Kopf vor: Kopf steht hier für einen Menschen, ein Teil steht für das Ganze, beide Entitäten liegen in der Erfahrungsdomäne ,Mensch'. In der neueren Forschung wird die Kontinuität der Übergänge zwischen Metonymie und Metapher hervorgehoben. Beide Mechanismen können im Usus auch interagieren, sich verzahnen, an den Schnittpunkten liegen. Die Interaktionsfälle bezeichne ich im Folgenden nach Goosens (2003) als Metaphtonymie.

\section{Mentales Bild und Motiviertheit der Phraseologismen}

Das Wesen der Metapher und Metonymie liegt darin, dass man zwei Entitäten konzeptuell verbindet, von denen die eine konkreter, d.h. besser vorstellbar als die andere, abstraktere ist. Dies geschieht auch in der Phraseologie: Die literale Lesart der Phraseologismen ist meistens konkret, anschaulich, auf dem Niveau der Alltagssprache angesiedelt und besteht aus oft gebrauchten Wörtern (vgl. dazu die Zusammenstellung der in der Phraseologie häufigsten Nomen von Quasthoff/ Schmidt/ Hallsteinsdóttir 2010: 39-41). Zu diesen alltäglich verwendeten Ausdrücken sind auf dem konzeptuellen (semantischen) Pol auf Grund der zahlreichen, alltäglichen Erfahrungen ausgebaute Wissensstrukturen in allen Modalitäten gesammelt. Einen prominenten Platz nehmen dabei wegen der Relevanz der visuellen Wahrnehmung visuelle mentale Repräsentationen ein. Aus kognitiv-psychologischer Sicht liegen Idiom-Konstituenten in der literalen Lesart also mehrheitlich auf einer Ebene, die Rosch et al. (1976) als Basisebene bezeichnen: Es ist eine 
Ebene, auf der die Kategorisierung durch ganzheitliche Wahrnehmung determiniert wird, auf der die Mehrheit unseres Wissens kategorisiert ist, mentale Bilder quasi automatisch generiert werden. Die Elemente der Basisebene werden von Probanden am schnellsten identifiziert und von den Kindern zuerst erworben. Als Begriffe der Basisebene werden beispielsweise: Apfel, Katze, Hammer eingestuft, im Gegensatz zu den Begriffen des höheren (Obst, Haustier, Werkzeug) und des niedrigeren (Jonagold, Perserkatze, Latthammer) Niveaus.

Die Natur der mentalen Bilder ist in der Phraseologie umstritten. Kontrovers diskutiert wird z.B. die Frage, ob die mentalen Bilder ausschließlich durch die literale Lesart evoziert sind, oder durch die metaphorischen oder metonymischen Mappings beeinflusst werden. Im erstgenannten Fall könnte das mentale Bild des zu besprechenden Idioms harter Brocken, eine Herausforderung' beispielshalber eine flüchtige Vorstellung eines trockenen Stücks Fleisch beinhalten, im zweitgenannten Fall könnte es das Bild einer Person sein, die sich an diesem Fleisch verschluckt. Cacciari/ Glucksberg (1990: 290) vertreten die Meinung, dass die meisten mentalen Bilder durch die literale Lesart des Idioms evoziert werden, Gibbs/ O'Brien (1990) propagieren die gegensätzliche Ansicht und verweisen darauf, dass die mentalen Bilder regelhaft durch konzeptuelle Metaphern beeinflusst werden und deswegen bei den meisten Menschen ähnlich sind. Generell ist anzumerken, dass die Frage nach der Natur von mentalen Bildern auf Grund methodologischer Schwierigkeiten unbeantwortet bleiben muss: Nach dem heutigen Wissensstand haben wir keine direkte Einsicht in dieses kognitive Phänomen und der indirekte Zugang über die Sprache ist eher problematisch: Die Versprachlichung von mentalen Bildern führt automatisch zu nachträglichen Interpretationen und eventuellen Verzerrungen, die in der natürlichen Kommunikation wegen ihrer Flüchtigkeit nicht vorauszusetzen sind. An dieser Stelle lasse ich also die komplizierte Problemstellung, inwieweit mentale Bilder Charakteristika vom idiomatischen Bild im Sinne von Burger (2010: 99) aufweisen, bestehen und versuche zu veranschaulichen, wie mentale Bilder, Metonymien und Metaphern bei der Konstituierung der aktuellen Bedeutungen (Konzeptualisierungen) mitspielen und wie sich aus diesen aktuellen Bedeutungen lexikalisierte Bedeutungen herausbilden.

\section{Empirische Untersuchung}

Das Idiom harter Brocken ist wegen der Ambiguität der nominalen Konstituente zur Untersuchung des Einflusses vom mentalen Bild auf Konzeptualisierungen besonders gut geeignet. Der Brocken als, unregelmäßig geformtes, oft von etwas abgebrochenes Stück' (DUW online, Zugriff am 3.08.2017) ist per se zu abstrakt, um ein mentales Bild auszulösen. Sobald man aber konkretisiert, wovon dieses Stück abgebrochen wurde, entstehen konsistente und kohärente mentale Bilder: Unter dem idiomatischen harten Brocken stellt man sich entweder einen harten Bissen, oder einen Felsenblock vor. 
Der semantischen Analyse wurden 40 per Zufallsgenerator zusammengestellten Belege aus den DWDS-Korpora ${ }^{2}$ unterzogen. Anhand der Untersuchung ließen sich folgende zwei Verwendungsprofile ermitteln: harter Brocken:

Verwendungsprofil 1: etw. ist ein harter Brocken (24 Belege), etw. stellt eine Herausforderung, eine schwierige Aufgabe dar ${ }^{6}$

Verwendungsprofil 2: $j d$. ist ein harter Brocken (16 Belege): 2.1. ,jd./ eine Mannschaft ist ein schwieriger Gegner' (11 Belege), 2.2. ,jd. ist robust, unnachgiebig' (5 Belege)

\section{Verwendungsprofil 1: etw. ist ein harter Brocken, etw. stellt eine Herausforderung, eine schwierige Aufgabe dar' (24 Belege)}

Die Motiviertheitsmechanismen sind für dieses Verwendungsprofil sehr interessant, weil das Idiom wenigstens zwei unterschiedliche mentale Bilder auslösen kann:

Der harte Brocken kann als ein Felsenblock konzeptualisiert werden, der einen Weg versperrt oder als ein hartes Stück von einer essbaren Sache, an dem man lange kauen muss. Diese zwei Interpretationen finden Bestätigung in dem markierten Gebrauch des Phraseologismus, in dem um das Idiom herum ein Szenario ausgebaut wird. Die metonymischen und metaphorischen Derivationen sind in beiden differente mentale Bilder voraussetzenden Fällen unterschiedlich.

\section{1. mentales Bild: ein großer, harter Happen, den man schwer schlucken kann}

(1) Das Berlin-Abkommen der Vier Mächte ist für die SED ein harter Brocken, den sie nur schwer schlucken kann. In der Wahlpropaganda spielt das Abkommen überhaupt keine Rolle, weil dies unangenehme Fragen der Bevölkerung provozieren könnte. Die Zeit, 29.10.1971, Nr. 44

(2) Der Handel mit für Vögel bestimmten Cannabissamen brachte Hanfhaus-Gründer vor Gericht. Wenn sie unter ihrem Futter Hanfsamen entdecken, dann picken viele gefiederte Freunde genussvoll zu. Was aber für Vögel ein besonderer Leckerbissen ist, kann für die Strafjustiz ein harter Brocken sein. Zwei Jahre wurde gegen das "Hanfhaus" ermittelt, weil dort angeblich unter der Tarnung von Vogelfutter mit Drogen gehandelt wurde. Der Tagesspiegel, 26.03.2001

(3) Es genügt nicht, die Weltbank, andere internationale Organisationen und Regierungen einzuspannen - auch die privaten Bankiers müssen mitziehen. Schließlich muß die Türkei noch einen besonders harten Brocken schlucken: Auf Betreiben Bonns versucht die Europäische Gemeinschaft als Gegenleistung den Aufschub für die Freizügigkeit türkischer Gastarbeiter, die vertragsgemäß bis 1986 weiter liberalisiert werden müßte. Die Zeit, 27.04.1979, Nr. 18

2 DWDS Zugriffsdatum: 7.7.2017, Suchanfrage: harter Brocken, Deutsches Textarchiv: 1/12, Kernkorpus 4/5, Die Zeit: 22/188, Berliner Zeitung: 8/89, Der Tagesspiegel: 5/55. 
Den Ausgangspunkt für die Konzeptualisierung bildet das mentale Bild, als weitere Motiviertheitsmechanismen sind Metapher und Metonymie anzusehen. Die Derivationsprozesse sind dabei auf zwei Ebenen angesiedelt. Auf der Ebene der Bedeutungsverschiebung der ganzen Wortverbindung kommen die konzeptuellen Metaphern SCHWIERIGKEITEN SIND UNGENIESS BARES ESSEN Sowie MIT ETW. FERTIG WERDEN IST ETWAS AUFESSEN UND VERDAUEN zum Vorschein. Die metaphorischen Mappings verbinden die mit der körperlichen Erfahrung zusammenhängende ESSEN-Domäne mit der abstrakteren Domäne der Bewältigung der Schwierigkeiten in der psychologischen Sphäre. In der Sprache findet man weitere Exemplifizierungen dieser konzeptuellen Metaphern, auch im phraseologischen Bereich:

Schwierigkeiten sind ungenießbares Essen

MIT ETW. FERTIG WERDEN IST ETWAS AUFESSEN UND VERDAUEN

etw. liegt mir auf dem Magen

etw. ist schwer zu verdauen

an etw. zu knabbern haben

sich an etw. verschlucken

eine harte Nuss für jmdn. sein, eine harte Nuss zu knacken haben, eine harte Nuss zu knacken bekommen

die Suppe auslöffeln (müssen), die man sich eingebrockt hat,

jmdm. die Suppe versalzen, jmdm. in die Suppe spucken

jmdm. das Leben sauer machen

sich (Dativ) die Zähne an etwas ausbeißen

in den sauren Apfel beißen (müssen)

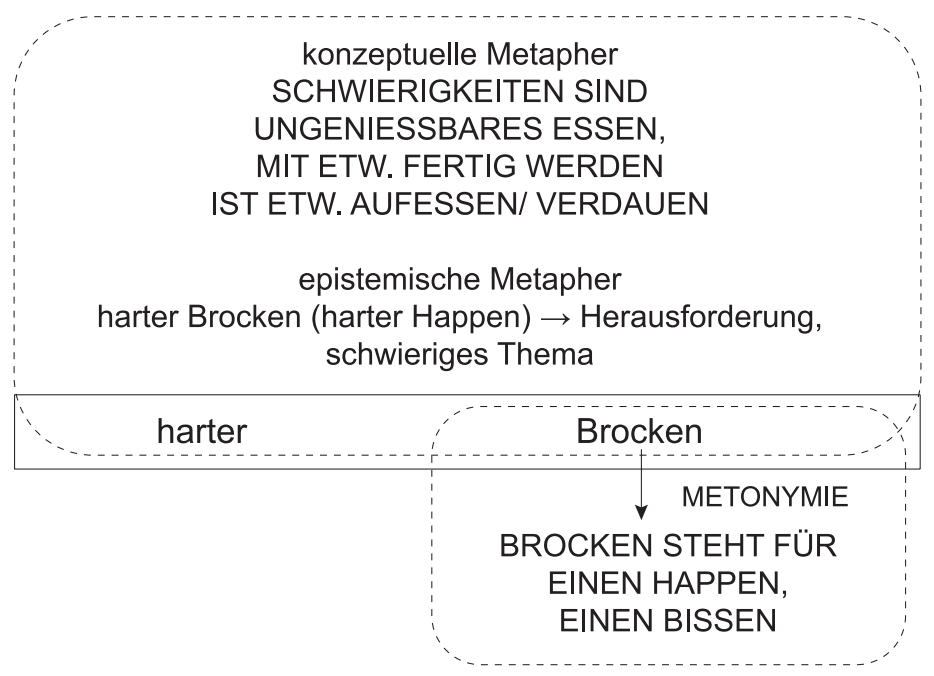

Abb. 1. Bedeutungskonstituierungsmechanismen für das erste

Verwendungsprofil mit dem mentalen Bild eines harten Happens, Bissens 
Auf der zweiten Ebene unterliegt nur die nominale Konstituente einer Bedeutungsderivation. Der Brocken steht hier metonymisch für einen Bissen, einen Happen. Schematisch lassen sich die Motiviertheitsmechanismen wie folgt darstellen:

\section{2. mentales Bild: ein großes, hartes, schweres Hindernis auf dem Weg}

Ein anderes mentales Bild liegt als Motiviertheitsbasis folgenden Belegen zugrunde:

(4) „Keiner soll für mich wählen, weil ich ein afrikanischer Amerikaner bin, niemand soll mir aber aus diesem Grund seine Stimme verwehren“, hieß damals Dinkins' Wahlspruch. Aber ausgerechnet aus dem „herrlichen Mosaik“ - eine Metapher für das New Yorker Völker- und Rassengemisch, die er während der Wahlkampagne so oft benutzte - sind ihm seit Amtsantritt die härtesten Brocken entgegengeflogen. Die Zeit, 20.07.1990, Nr. 30

(5) Denn in der Tat haben Deutschlands Olympiafahrer beim Kampf um die Zuschauergunst harte Brocken aus dem Weg zи räumen - und ein unüberwindlicher wird ihnen ständig im Wege sein. Das ist die Zeitverschiebung, die die Fernsehbetrachtung von Finalläufen in die frühesten Morgenstunden verlegt. Der Tagesspiegel, 11.09.2000

(6) Der Druck, den die Amerikaner jetzt machen, wird Scharping gegen die Sparer helfen. Der Rest ist Hoffnung - auf die Konjunktur, auf den Erfolg des Sparprogramms, auf die nächste Wahl. Wer will dann Standorte schließen? Einstweilen kann sich Rudolf Scharping an den harten Brocken noch vorbeidrücken. Die Zeit, 13.07.2000, Nr. 29

Die Verben bzw. verbale Phraseologismen entgegenfliegen, aus dem Weg räumen, sich vorbeidrücken verweisen darauf, dass der harte Brocken hier in der zweiten Auslegung, d.h. als ein Felsenblock, ein Stein, ein Klotz konzeptualisiert wird. In diesem Fall geht man also auch von anderen konzeptuellen Metaphern und Metonymien aus:

Grundlegend für die Konzeptualisierung ist die konzeptuelle Metapher SCHWIERIGKEITEN SIND HINDERNISSE AUF DEM WEG, die sich in die weit verbreitete Weg-Metaphorik einfügt. Die konzeptuelle Metapher LEBEN IST EIN WEG stellt nämlich ein plakatives Beispiel einer konzeptuellen Metapher von einem großen Generalitätsgrad dar. Diese Metapher beruht auf dem in der körperlichen Erfahrung fest verankerten image schema ${ }^{3}$ PATH und ist in der Alltagssprache so allgegenwärtig (vgl. z.B. Lebenslauf, Lebensweg), dass der metaphorische Charakter der Konzeptualisierungen öfters gar nicht auffällt: Es ist a metaphor we live by, wie es in dem Titel der bahnbrechenden Publikation von Lakoff/ Johnson (1980) steht. Die Ausgangsdomäne bildet eine Vorstellung einer Wegstrecke, die man nach und nach bewältigt. Diese relativ konkrete Vorstellung wird auf abstrakteren Bereich des zeitlichen Verlaufs des Lebens übertragen: Das menschliche Leben - sowohl in der Bedeutung eines Lebensweges als auch eines geistigen Entwicklungsprozesses (vgl.

${ }^{3}$ Unter image schemas verstehe ich hier nach Johnson (1987) präkonzeptuelle, körperlich verankerte räumlich-analoge Vorstellungsschemata. 
geistig Zurückgebliebene) - wird als ein Weg konzeptualisiert (seinen Weg gehen/ machen), das durch die Berge und Täler (bergauf, bergab gehen, nicht ganz auf der Höhe sein) führt, gerade oder gewunden, bequem oder unbequem ist (ein steiniger Weg, sich auf einem schwankenden Boden bewegen). Vor dem Hintergrund dieser konzeptuellen Metapher werden die Geburt (auf die Welt kommen), der Tod (jmds. letzter Weg, den letzten Weg gehen), Freundschaft und Liebe (jmds. Wege kreuzen sich), Trennung (auf dem Scheideweg stehen), Betrug (einen Seitensprung machen), Karriere (es weit bringen), Lebensfehler (sich selbst den Weg verbauen), Konkurrenz (auf der Strecke bleiben, nicht mitkommen) konzeptualisiert. Als eine Metapher von einem niedrigeren Generalitätsgrad, die durch die Weg-Metaphorik motiviert ist, kann die Metapher SCHWIERIGKEITEN SIND HINDERNISSE AUF DEM WEG angesehen werden, die sich beispielshalber in folgenden Phraseologismen manifestiert:
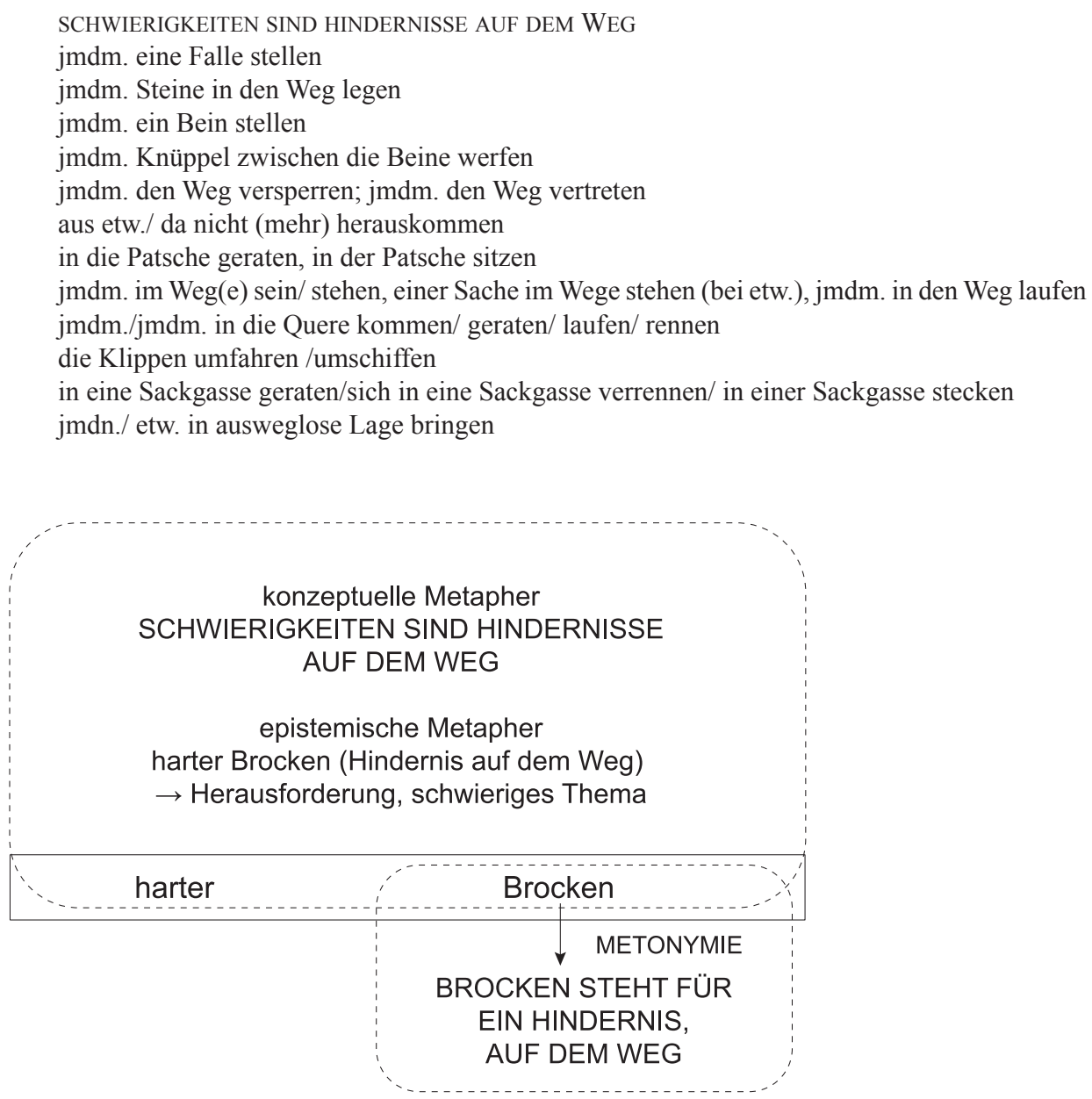

Abb. 2. Bedeutungskonstituierungsmechanismen für das erste Verwendungsprofil mit dem mentalen Bild eines Hindernisses 
Einer Bedeutungsderivation unterliegt auch die nominale Konstituente: Der Brocken steht hier metonymisch für ein großes Hindernis auf dem Weg in der Form eines Findlings, Felsenblocks u.Ä., das aus dem Weg geschafft oder umgangen werden muss.

Die dargestellten Belege stellen einen Beweis für die Relevanz des mentalen Bildes als eines Motiviertheitsmechanismus dar. Die Grundzüge des mentalen Bildes lassen sich auf Grund der kontextuellen Umgebung und des ausgebauten Szenarios relativ eindeutig identifizieren, die metonymischen und metaphorischen Derivationen sind an das Vorstellungsbild aufs Engste gebunden. Nicht in allen Belegen ist das Idiom dennoch markiert gebraucht, in den meisten Fällen stehen dem Rezipienten beide Interpretationsmöglichkeiten mit subtilen Bedeutungsdifferenzen zur Verfügung:

(7) Die Deutsche Telekom will die Gehälter der in die konzerneigene Beschäftigungsagentur ausgelagerten Mitarbeiter senken und stößt dabei auf massiven Widerstand der Gewerkschaft. „Dieser Punkt wird der härteste Brocken in unseren Verhandlungen“, heißt es in Kreisen des Telekom- Betriebsrates und der Gewerkschaft Verdi. „Eine Entgeltreduzierung ist eigentlich in den unteren Tarifgruppen inakzeptabel. Der Tagesspiegel, 30.01.2004

(8) „Beliebt bei unseren Kunden, gehaßt bei unserer Konkurrenz“, so stellt ein Unternehmen sein Angebot per Anzeigen der Öffentlichkeit vor. Der „harte Brocken für die Konkurrenz“ - so wörtlich in einer Anzeige - sind First Class Hotelappartements an der spanischen Sonnenküste. Mit mehr als zehn Prozent jährlicher Mindestrendite will man deutsche Käufer locken. Die Zeit, 23.04.1971, Nr. 17

In beiden Gebrauchsbelegen kann der harte Brocken als ein Hindernis, das umgangen bzw. aus dem Weg geräumt werden muss, interpretiert werden. Der Brocken ist groß, so wie die zu bewältigenden Verhandlungspunkte bzw. Aufgaben für die Konkurrenz relevant und schwer sind. Sein Wegräumen nimmt viel Zeit und Mühe in Anspruch (harte Gesteine haben eine entsprechende Dichte und ein erhebliches Gewicht) - damit müssen aber auch Verhandlungsführer oder Manager in den sozial-ökonomischen Zieldomänen rechnen. Genauso wahrscheinlich ist aber auch die Konzeptualisierung des harten Brockens als harten Bissens. Ein hartes Stück Essen kann auf zweierlei Arten zu sich genommen werden: Entweder knabbert man lange daran, kaut es gründlich, was viel Zeit und Geduld erfordert, oder man versucht es auf einmal zu schlucken, was jedoch Verschluckungsgefahr und Verdauungsprobleme mit sich zieht. Diese interpretativen Spielräume sind auch in den Zieldomänen vorhanden: Es handelt sich jeweils um schwierige Angelegenheiten, die man entweder länger zeit- und arbeitsaufwändig bearbeitet oder in einem entschiedenen, dennoch riskanten Schritt löst (indem der Vorstand seinen Willen ungeachtet der Proteste durchsetzt oder die Unternehmen ihre Immobilienpreise stark senken). Verbindet man die beiden Domänen, dann entstehen epistemische Mappings als ein zusätzlicher Motiviertheitsmechanismus. Schematisch lassen sich die Bedeutungskonstituierungsmechanismen wie folgt darstellen: 


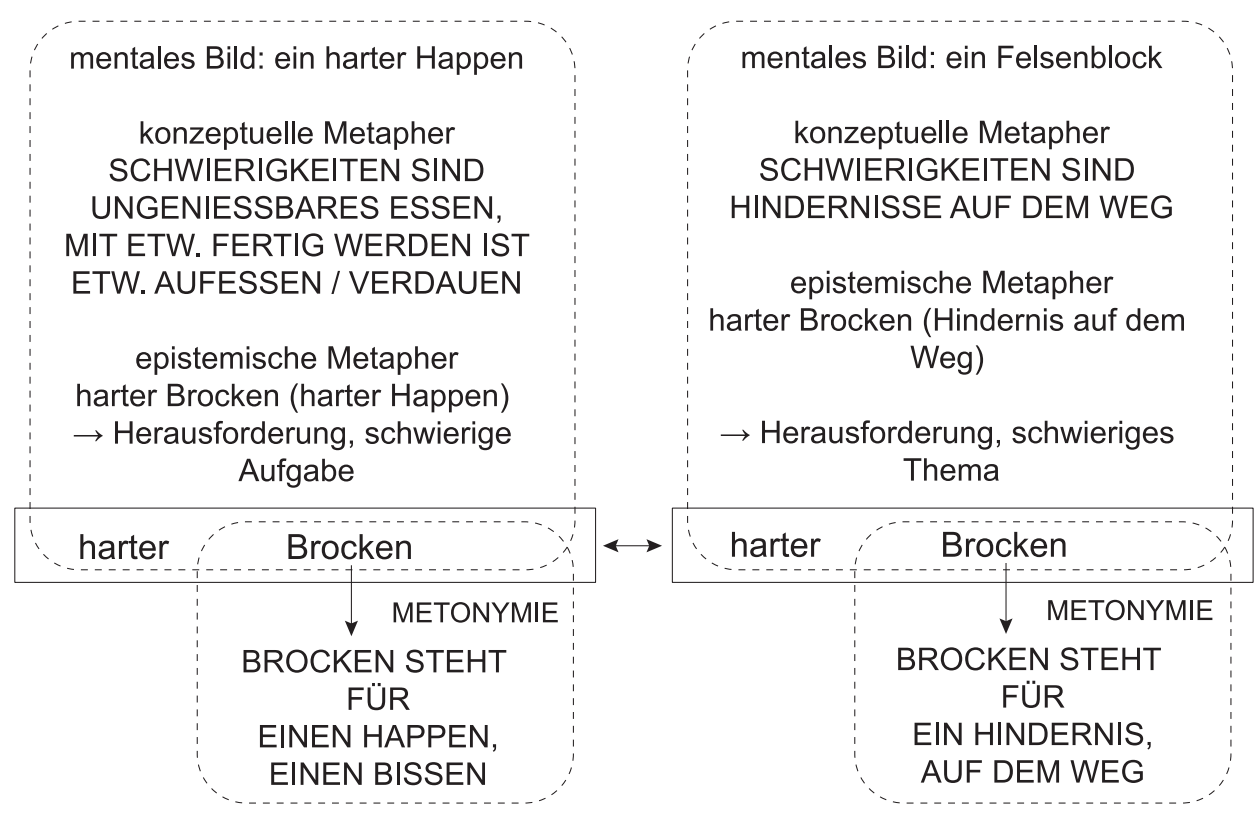

Abb. 3. Bedeutungskonstituierungsmechanismen für das erste Verwendungsprofil bei einem unmarkierten Gebrauch des Idioms

\section{Verwendungsprofil 2: jd./ eine Mannschaft ist ein harter Brocken ,ein schwieriger Gegner, eine unnachgiebige, robuste, zielstrebige Person/ Personengruppe' (16 Belege)}

Die Bedeutungskonstituierungsmechanismen sind für dieses Verwendungsprofil schwerer modellierbar, als es im ersten Verwendungsprofil der Fall war. Das Idiom bezieht sich in diesen Verwendungsprofilen auf belebte Subjekte, kommt dabei kaum markiert vor, so dass die Frage, ob die nominale Konstituente Brocken überhaupt näher bestimmt wird und das mentale Bild evoziert, offen bleibt. Nichtsdestotrotz ist die Verbindung zwischen der literalen und der phraseologisierten Lesart für die meisten Sprachteilhaber nachvollziehbar, das Idiom ist motiviert. Zwei Verwendungsmuster lassen sich ermitteln, die in verschiedenen Diskursbereichen gebraucht werden.

\section{1. (Sport) jd./ eine Mannschaft ist ein harter Brocken (11 Belege)}

Beide mentalen Bilder können in elf Belegen evoziert werden, in denen das Idiom im Diskursbereich Sport auf einen schwierigen, schwer zu besiegenden Gegner referiert, z.B.:

(9) "Das nimmt in den letzten zwei Gruppenspielen ein wenig den Druck aus den Partien. Trotzdem wollen wir nun natürlich auch Gruppensieger werden, um erst möglichst spät auf die 
harten Brocken aus den anderen Gruppen zu treffen". Düsseldorf kann nach vier Siegen in der Gruppe D nicht mehr von einem der ersten beiden Plätze verdrängt werden. Die Zeit, 21.11.2014 (online)

(10) Darüber hinaus hatte Bastian Schweinsteiger seine Erkältung, die ihn unter der Woche zur einer Trainingspause gezwungen hatte, noch rechtzeitig auskuriert. Von Beginn an zeichnete sich ab, dass die Serben ein härterer Brocken sein würden als Australien. Löw hatte seine Mannschaft im Vorfeld auch eindringlich vor den Serben gewarnt, nachdem diese ihren WM-Auftakt gegen Ghana (0:1) verpatzt hatten. Die Zeit, 18.06.2010 (online)

Wie erwähnt, sind die ausgebauten Szenarios, die auf das mentale Bild schlussfolgern ließen, in diesem Verwendungsprofil nicht vorhanden. Als ein leichter Hinweis auf die Präferenz für das mentale Bild eines wegen seiner Härte schwer zu knabbernden Happens können Komparativformen der adjektivischen Konstituente in zwei Belegen dieses Verwendungsprofils (vgl. stellvertretend Beleg 10) gedeutet werden: Die größere Härte eines Happens in der Ausgangsdomäne dürfte hier als ein erhöhter Schwierigkeitsgrad, ein größeres Risiko einer Niederlage gedeutet werden. Die kognitiven Mechanismen der Bedeutungskonstituierung in den übrigen 9 Belegen sind dennoch schwankend und lassen sich nicht eindeutig festlegen.

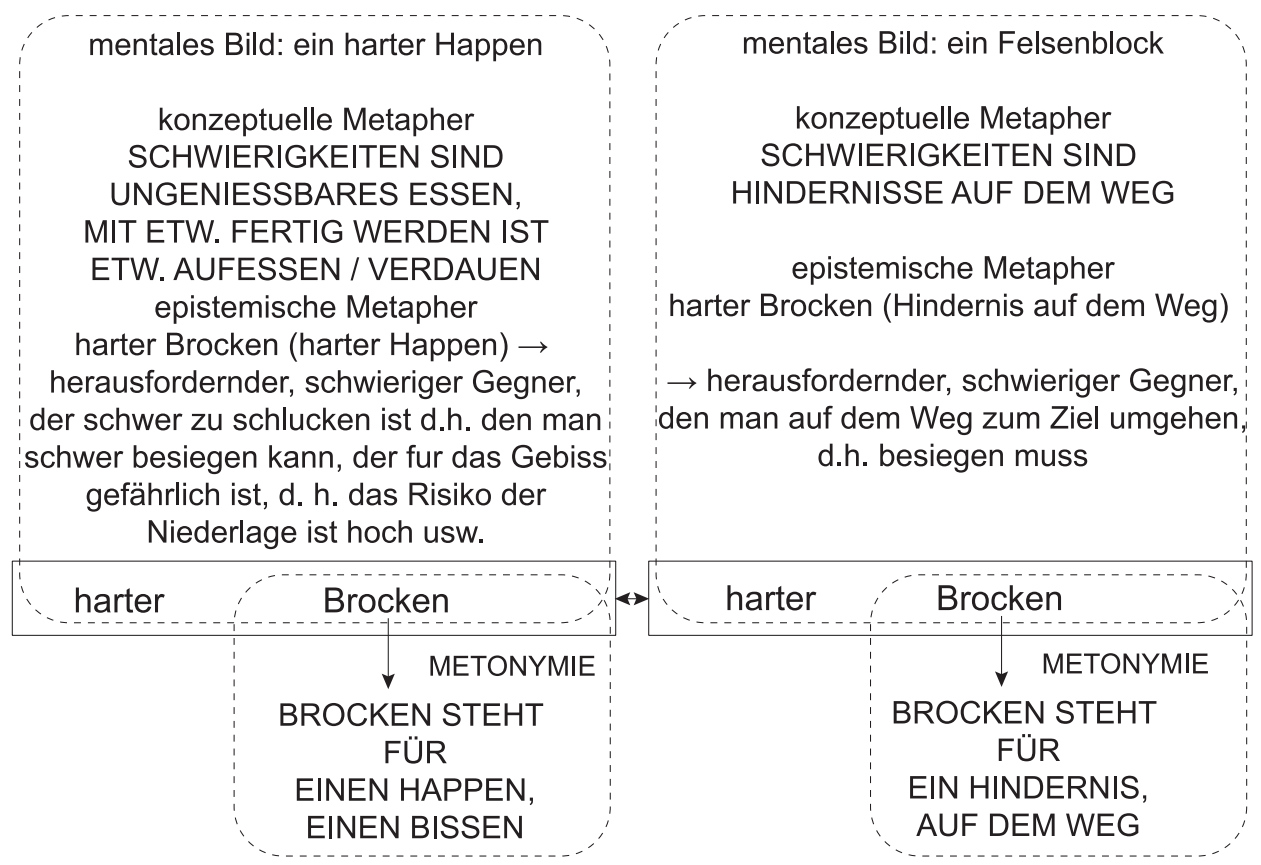

Abb. 4. Bedeutungskonstituierungsmechanismen für das Verwendungsprofil 2.1. 


\section{2. jd. ist ein harter Brocken (5 Belege)}

In den letzten fünf Belegen referiert harter Brocken auf robuste, unnachgiebige Personen, die schwer im Umgang sind: Hervorgehoben wird der starke Charakter, die Rücksichtslosigkeit dieser Menschen, ihre Zielstrebigkeit, Widerstandsfähigkeit, Resistenz gegen äußere Einflüsse (im Polnischen existiert das Idiom twarda sztuka, im Englischen a tough cookie).

(11) In der Männerwelt des Militärs setzt sie ihre unerbittlichste Maske auf und stellt an ihrer Uniform Fallschirmspringer-Marke sowie Gefechtsabzeichen aus. »Ich etabliere mich erst als harter Brocken und verhalte mich dann weicher«, sagt sie. Die Zeit, 09.06.2004, Nr. 25

(12) Man brauchte kein Psychologe zu sein, um zu erkennen, daß Haerynk ein ungleich härterer Brocken für kriminalpolizeiliche Vernehmungsversuche war. »Es ist zu bedenken«, hatte der Kriminalbeamte Lange in einem Aktenvermerk vom 27.9.1971 niedergelegt, »daß es sich bei Haerynk um einen mehrfach vorbestraften Rechtsbrecher handelt, welcher nur etwas zugibt, was man ihm auch beweisen kann«. Hannover, Heinrich: Die Republik vor Gericht 19541974, Berlin: Aufbau-Taschenbuch-Verl. 2001 [1998], S. 461

In diesem Verwendungsmuster scheint die Konzeptualisierung des Brockens als ein Hindernis auf dem Weg unwahrscheinlich zu sein. Harter Brocken wird eher als ein hartes Stück Essen, an dem lange gekaut werden muss, konzeptualisiert, die motivierenden Verbindungen sind dann auf die bereits beschriebenen konzeptuellen Mappings: SCHWIERIGKEITEN SIND UNGENIESSBARES ESSEN, MIT ETW. FERTIG WERDEN IST ETW. AUFESSEN/VERDAUEN sowie die epistemischen Mappings zurückzuführen:

Tab. 1. Epistemische Mappings für das Verwendungsprofil 2.2.

\begin{tabular}{|l|l|}
\hline \multicolumn{1}{|c|}{ Harter Brocken (source) } & Starke, widerstandsfähige Person (target) \\
\hline hartes Stück, das man schwer (ab-)bricht & abgehärtete Person mit starkem Charakter, \\
& den man schwer brechen, d.h. unterordnen \\
& kann \\
schwer zu knabbern & eine Person, die man nur schrittweise für \\
& etw. gewinnen kann \\
langes Kauen & viel Anstrengung und Aufmerksamkeit im \\
& Umgang notwendig \\
gefährlich für das Gebiss & hohes Risiko der Niederlage, wenn man \\
& ungekonnt handelt \\
\hline
\end{tabular}

Möglich ist aber auch, dass der Brocken in diesen Konzeptualisierungen nicht näher festgelegt wird und kein konsistentes mentales Bild hervorruft. Die phraseologische Motiviertheit ergibt sich dann aus den Bedeutungsderivationen beider Konstituenten: Der Brocken steht dann metonymisch für einen Menschen. Diese metonymische Verschiebung ist übrigens z.T. lexikalisiert, vgl. die zweite Teilbedeutung im DUW: ugs. ,dicke, massige Person'. Lexikalisiert ist ebenfalls die metaphtonymische Derivation: HÄRTE STEHT FÜR STRENGE/STRENGE IST WIE 
HÄRTE. (vgl. die dritte Teilbedeutung des Adjektivs hart 3.a ,ohne Mitgefühl; unbarmherzig, streng', 3.b ,(von jemandes Äußerem) nicht mild, empfindsam, weich, sondern scharf und streng ${ }^{6}$ ). Härte als eine prototypische Eigenschaft von Steinen und Metallen wird mit Unnachgiebigkeit, Resistenz, schwieriger Bearbeitung assoziiert, diese Eigenschaften werden dann auf die Eigenschaften des Körpers (harte Augen) und der Psyche übertragen (harter Kerl, ein hartes Herz haben, harte Schule). Die Bedeutungskonstituierungsmechanismen für dieses Verwendungsmuster, die zwischen beiden Interpretationen schwanken, stellt Abb. 5 dar:

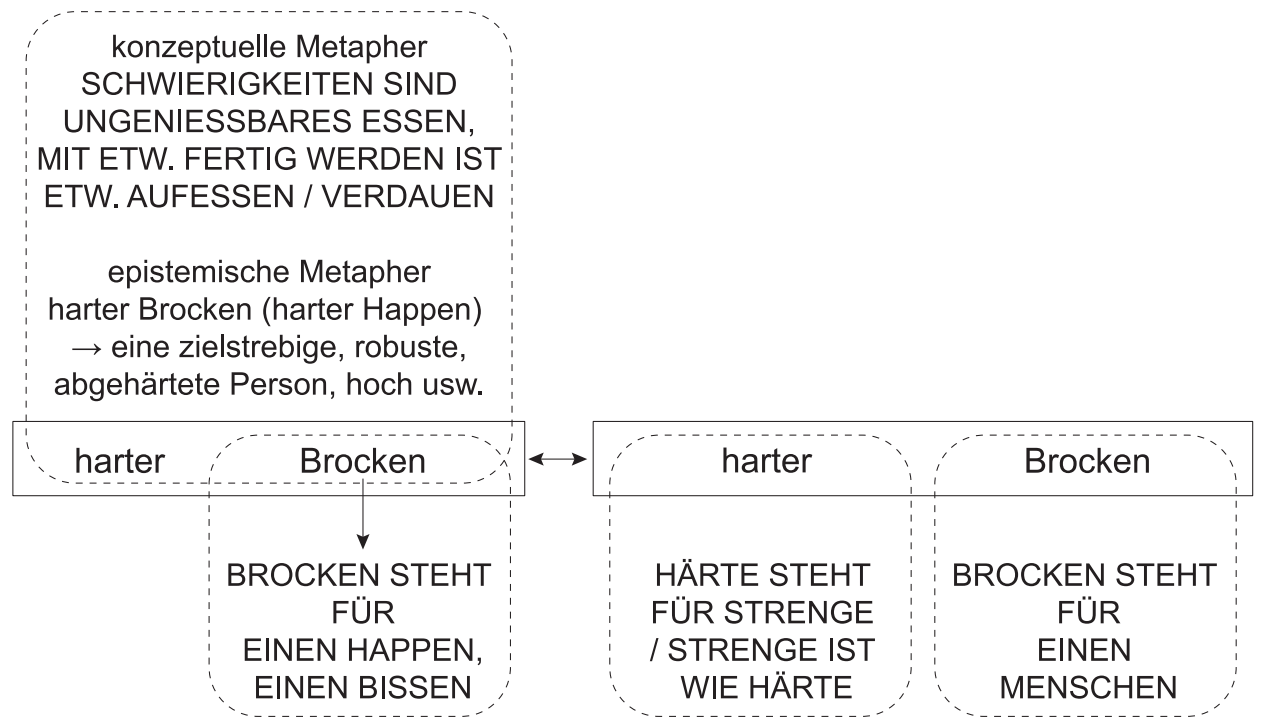

Abb. 5. Bedeutungskonstituierungsmechanismen für das Verwendungsprofil 2.2.

\section{Zusammenfassung}

Die durchgeführte Untersuchung veranschaulicht die Vielfalt und Komplexität der Bedeutungskonstituierungs- und Motiviertheitsmechanismen. Die Relevanz der metaphorischen und metonymischen Derivationen ist allgemein anerkannt, wurde in mehreren Veröffentlichungen behandelt (vgl. z.B. Feyaerts 1999; Sulikowska 2015; Szczęk 2010) und spiegelt sich sogar in der Terminologie wider, vgl. z.B. die Metaphernphraseme bei Rothkegel (2014: 275) oder metaphorische Idiome bei Burger (2010: 88). Die vorliegende Analyse verweist auf den richtungsweisenden Beitrag des mentalen Bildes auf die Entstehung der Bedeutungen und ihre nachträgliche Interpretierbarkeit. Die stark von der literalen Lesart beeinflussten mentalen Bilder eröffnen Spielräume, in denen Interpretationen möglich sind. Erst in diesen Spielräumen verlaufen dann semantische Derivationen, denen 
konzeptuelle Metaphern und Metonymien zugrunde liegen, die später durch epistemische Mappings ergänzt werden. Zweifelsohne beeinflussen mentale Bilder den emotionalen Konnotationswert der Phraseologismen und erhöhen ihre semantische Mehrdeutigkeit und Ambiguität: So wie die materiellen Bilder von Natur aus mehrdeutig und polyvalent sind (Stöck1 2004: 151), so erweitern auch mentale Bilder die Interpretationsmöglichkeiten der idiomatischen Bedeutung und bewirken, dass ihre Modellierung komplex und vielschichtig ist.

\section{Literatur}

Bally, Charles (1905): Précis de stylistique: esquisse d'une methode fondée sur l'étude du français moderne. Genève.

Barcelona, Antonio (2003): Clarifying and applying the notions of metaphor and metonymy within cognitive linguistics: An update. In: Dirven, René / Pörings, Ralf (Hrsg.): Metaphor and Metonymy in Comparison and Contrast. Berlin/New York. S. 207-278.

Barsalou, Lawrence W. (1999): Perceptual symbol systems. In: Behavioral and Brain Sciences 22. S. 577-660.

Barsalou, Lawrence W. (2012): The Human Conceptual System. In: Spivey, Michael J. / Mcrae, Ken / Joanisse, Marc F. (Hrsg.): The Cambridge Handbook of Psycholinguistics. Cambridge. S. 239-258.

Burger, Harald (2007): Semantic aspects of phrasemes. In: Burger, Harald / Dobrovol'skij, Dmitrij / Kühn, Peter / Norrick, Neal R. (Hrsg.): Phraseologie - Phraseology. Ein internationales Handbuch der zeitgenössischen Forschung. Bd. 1. Berlin/New York. S. 90-110.

Burger, Harald (2010): Phraseologie. Eine Einführung am Beispiel des Deutschen. Berlin.

Cacciari, Cristina / Glucksberg, Sam (1975): Understanding idioms: Do visual images reflect figurative meanings? In: European Journal of Cognitive Psychology 7:3. S. 283-305.

Černyševa, Irina (1980): Feste Wortkomplexe des Deutschen in Sprache und Rede. Moskva.

Croft, William (2003): The role of domains in the interpretation of metaphors and metonymies. In: Dirven, René / Pörings, Ralf (Hrsg.): Metaphor and Metonymy in Comparison and Contrast. Berlin/New York. S. 161-206.

Feyaerts, Kurt (1999): Die Metonymie als konzeptuelles Strukturprinzip: eine kognitiv-semantische Analyse deutscher Dummheitsausdrücke. In: Baur, Rupprecht S. / Chlosta, Christoph / Piirainen, Elisabeth (Hrsg.): Wörter in Bildern - Bilder in Wörtern. Beiträge zur Phraseologie und Sprichwortforschung aus dem Westfälischen Arbeitskreis. Baltmannsweiler. S. 139-176.

Gibbs, Raymond W. / O’Brien, Jennifer E. (1990): Idioms and mental imagery: The metaphorical motivation for idiomatic meaning. In: Cognition 36. S. 35-68.

Johnson, Mark (1987): The Body in the Mind: The Bodily Basis of Meaning, Imagination, and Reason. Chicago.

Kövecses, Zoltan (2002): Metaphor. A practical introduction. New York.

Lakoff, George (1993): The Contemporary Theory of Metaphor. In: Ortony, Andrew (Hrsg.): Metaphor and Thought. Cambridge. S. 202-251.

Lakoff, George / Johnson, Mark (1980): Metaphors we live by. Chicago.

Langacker, Ronald W. (1987): Foundations of Cognitive Grammar. Vol. 1. Theoretical Prerequisites. Stanford.

Langacker, Ronald W. (2008): Cognitive Grammar. A Basic Introduction. Oxford.

Palm, Christine (1995): Phraseologie. Eine Einführung. Tübingen.

Germanica Wratislaviensia 143, 2018

(C) for this edition by CNS 
Poddig, Frank (1995): Die Enkodierung und Dekodierung piktorieller Werbebotschaften: ein semiotisch-kognitiver Ansatz. Wiesbaden.

Quasthoff, Uwe / Schmidt, Fabian / Hallsteinsdóttir, Erla (2010): Häufigkeit und Struktur von Phraseologismen. In: Ptashnyk, Stefaniya / Hallsteinsdóttir, Erla / Bubenhofer, Noah (Hrsg.): Korpora, Web und Datenbanken. Computergestützte Methoden in der modernen Phraseologie und Lexikographie. Baltmannsweiler. S. 37-54.

Rickheit, Gert / Weiss, Sabine / Eikmeyer, Hans-Jürgen (2010): Kognitive Linguistik. Theorien, Modelle, Methoden. Tübingen/Basel.

Rosch, Eleanor / Merrvis, Carolyn / Gray, Wayne / Johnson, David / Boyes-Braem, Penny (1976): Basic Objects in Natural Categories. In: Cognitive Psychology 8. S. 382-439.

Rothkegel, Annely (2014): Sprachliche Bilder und Modelle: Bedeutungskonstruktion abstrakter Begriffe durch Metaphernphraseme (Beispiele GESUNDHEIT UND RISIKO). In: Germanica Wratislaviensia 139. S. 275-289.

Stöckl, Hartmut (2004): Die Sprache im Bild - Das Bild in der Sprache. Zur Verfassung von Sprache und Bild im massmedialen Text. Berlin/New York.

Sulikowska, Anna (2015): Zur Motiviertheit der Idiome am Beispiel des Phraseologismus, ein heißes Pflaster'. In: Colloquia Germanica Stetinensia 24. S. 171-189.

Szczęk, Joanna (2010): Auf der Suche nach phraseologischer Motiviertheit im Deutschen (am lexikographischen Material). Dresden/Wrocław.

DWDS $=$ Digitales Wörterbuch der Deutschen Sprache, www.dwds.de (Zugriff am 1-22.07.2017).

DUW $=$ Duden Universalwörterbuch, www.duden.de (Zugriff am 1-22.07.2017). 